\title{
Magnon excitations in vortex-state nanorings
}

\author{
C.E. Zaspel ${ }^{1}$, J.W. Owens ${ }^{1}$, and B.A. Ivanov ${ }^{2}$ \\ ${ }^{1}$ Department of Environmental Sciences, University of Montana-Western, Dillon, MT 59725, USA \\ ${ }^{2}$ Institute of Magnetism, National Academy of Sciences of Ukraine, Kiev 03412, Ukraine \\ E-mail: bivanov@i.com.ua
}

Received March 13, 2006, revised April 4, 2006

\begin{abstract}
The magnon mode excitation spectrum is calculated using the linearized Landau-Lifshitz equations applied to the ring in the vortex ground state. A combination of analytical techniques with numerical evaluation of integrals is used to obtain the mode frequency with the effective magnetostatic volume charge as a small perturbation. In general, the modes can be classified according to radial number $n$ and azimuthal number $m$. It is shown that the frequency increases as $n$ increases, and the frequency decreases as $m$ increases.
\end{abstract}

PACS: 75.75.+a, 75.30.Ds, 75.40.Gb

Keywords: Landau-Lifshitz equation, magnetostatic interaction, exchange energy, exchange length, vortex state, nanoring, radial number, azimuthal number.

Magnetic microdots made from a soft ferromagnetic material such as Permalloy have been proposed [1] for use as magnetic storage media, and the study of the basic physics of magnetic particles in this size range has led to interesting effects. For example, when the radius is reduced to the micron and submicron range the competition between exchange and the magnetostatic interaction will result in a vortex ground state with the magnetization mainly confined to the plane. This structure is stable because the curling effect in the planar vortex will eliminate effective magnetostatic charges, thereby minimizing the energy. However, at the core of the planar vortex there is a high exchange energy singularity, which is eliminated by an out-of-plane rotation of the magnetization at the core, resulting in a small surface magnetic poles (magnetic charges), which has also been observed [2] in thin dots. In materials such as Permalloy the presence of the core results in a net magnetic moment perpendicular to the plane over a central region of about $20 \mathrm{~nm}$ diameter. If arrays of dots are to be used in magnetic information storage, this vortex core presents several disadvantages. The net magnetic moment of the will interact through the dipolar interaction with other dots in the array, affecting properties such as switching of the curling direction. Because of the presence of the core, there is a low frequency
(subGHz) gyrotropic mode [3] that will complicate the dynamics of the single as well as the array of dots. Finally, the vortex state is only stable above a critical diameter of approximately $100 \mathrm{~nm}$, limiting the possible bit density in arrays of these systems.

Some of these problems can be overcome by the use of a ring rather than a disk, which also has a vortex ground state [4-6]. Owing to the geometry of the ring there is no core, and therefore no magnetostatic fields other than those from fluctuations about the vortex ground state. In addition, the vortex state is stable for smaller diameters than the dot resulting in a higher potential bit density for an array of rings. In order for the ring vortex state to be useful, it is necessary to develop a simple method to produce the vortex state and switch the curling direction of this state, which was recently [4-6] shown to be accomplished by an inplane magnetic field pulse. More recently switching was accomplished using a spin-polarized current pulse [7] rather than an external magnetic field.

When considering the response of a ring vortex to a pulse is desirable to have an understanding of the dynamic properties of the vortex state. In the disk, there has already been both theoretical and experimental research that has advanced the understanding of vortex dynamics in this geometry. In general, the linearized Landau-Lifshitz equations are solved [8-10] with the 
appropriate disk boundary conditions with both exchange and magnetostatic interactions to obtain the magnon mode frequencies. For the vortex state disk the lowest frequency subGHz gyrotropic mode as well as a rich spectrum of higher frequency modes have been observed [3,11] in experiments such as time-resolved Kerr microscopy as predicted by various solutions of the Landau-Lifshitz equations. For the case of rings, both experimental and theoretical studies are lacking except for ferromagnetic resonance [12] done with an array of rings. For this reason, in the following the magnon mode frequencies are calculated on the vortex ground state. This is done using the vortex-magnon interaction including magnetostatic effects as was previously done for the vortex state where the core [13] was included. It is remarked that the modes are in the $\mathrm{GHz}$ range for all modes, and the lower frequency subGHz gyrotropic mode does not appear owing to the absence of the vortex core.

We begin by considering small oscillations of the magnetization about the vortex ground state with the magnetostatic field included. The dynamic properties are determined by the Landau-Lifshitz equation, which is

$$
\frac{\partial \mathbf{M}}{\partial t}=\gamma \cdot \mathbf{M} \times \frac{\delta W}{\delta \mathbf{M}},
$$

where $W=W[\mathbf{M}]$ is the energy functional, $\gamma=g \mu_{B} / \hbar, g \approx 2$ is the gyromagnetic ratio, and $\mu_{B}$ is the Bohr magneton. The magnetization is expressed as $\mathbf{M}=M_{S}(\sin \theta \cos \varphi, \sin \theta \sin \varphi, \cos \theta)$ where $\theta$ is the polar angle relative to the ring symmetry axis, and $\varphi$ is the azimuthal angle. First it is assumed that the magnetization is uniform along the ring axis $z$. For the ring in the vortex ground state the angular variables can be written in terms of polar coordinates $(r, \chi)$ in the plane as

$$
\theta=\pi / 2+\theta(r, \chi, t), \varphi=\chi+\pi / 2+\psi(r, \chi, t),
$$

where $\theta, \psi$ describe the small oscillations on the ground of the magnetic vortex.

The energy of the vortex state contains contributions from both isotropic ferromagnetic exchange and the magnetic dipolar interaction. In the continuum approximation these two contributions to the energy density can be written as an inhomogeneous isotropic exchange term and an effective magnetostatic field,

$$
w(\nabla \mathbf{M}, \mathbf{M})=\frac{A}{2 M_{s}^{2}}(\nabla \mathbf{M})^{2}-\frac{1}{2} \mathbf{M} \mathbf{H}_{m},
$$

where $\mathbf{M}$ is the magnetization, $M_{s}$ is the saturation magnetization, and the vortex energy is obtained by integration of the energy density over the ring volume:

$$
W=\int w(\nabla \mathbf{M}, \mathbf{M}) d^{3} x .
$$

The first term in Eq. (3) is the contribution from the exchange interaction, which is short-range and local in nature, and the second term contains the magnetostatic field $\mathbf{H}_{m}$. The sources of this field are both volume charges arising from $\nabla \cdot \mathbf{M}$ and surface charges from the normal component of $\mathbf{M}$ at the surface. The out-of-plane components of magnetization produce the charges on upper and lower surfaces, having the form $\mathbf{H}_{m}=-4 \pi \hat{\mathbf{z}} \cos \theta$, which are local, and other contributions have the form of nonlocal fields obtained from the potential, $\mathbf{H}_{m}=-\nabla \Phi$. The potentials from the volume and edge surface magnetostatic charges are known to be,

$$
\begin{gathered}
\Phi_{v}=\int \frac{\nabla \cdot \mathbf{M}\left(\mathbf{r}^{\prime}\right)}{\left|\mathbf{r}-\mathbf{r}^{\prime}\right|} r^{\prime} d r^{\prime} d \chi^{\prime} d z^{\prime}, \\
\Phi_{s}= \pm R_{i . o} \int \frac{\hat{\mathbf{r}} \cdot \mathbf{M}\left(R_{i, o}\right)}{\left|\mathbf{r}-R_{i, o} \hat{r}\right|} d \chi^{\prime} d z^{\prime},
\end{gathered}
$$

where

$\left(\mathbf{r}-\mathbf{r}^{\prime}\right)^{2}=r^{2}+r^{\prime 2}-2 r r^{\prime} \cos \left(\chi-\chi^{\prime}\right)+\left(z-z^{\prime}\right)^{2}$,

the upper sign is used at the inner edge $R_{i}$ and the lower sign is used at the outer edge $R_{o}$. The other parameter $L$ is the ring thickness, and the ring radius satisfies the condition $R_{o} \gg L$. Finally, the energy can be expressed as a sum of contributions from exchange, edge, and volume charges, $W=W_{\mathrm{ex}}+$ $+W_{e}+W_{v}$.

The edge field, being formally proportional to the small parameter $L / R_{i, o}$, has singular behavior near the edges, and rapidly decays as $R_{i, o} /\left(R_{i, o}-r\right)$ for $R_{i, o}-r \gg L$. Thus, the edge magnetostatic field is sharply peaked at the edge, and it gives the effective boundary condition [13]. To obtain the concrete form of the boundary condition for the ring geometry it is first necessary to calculate the magnetostatic energy arising from the effective magnetostatic charge at the ring edges. We begin with the key ansatz for the functions $\theta$ and $\psi$, see Eq. (2)

$$
\begin{aligned}
& \theta(r, \chi, t)=f(r) \cos (m \chi+\omega t), \\
& \mu(r, \chi, t)=g(r) \sin (m \chi+\omega t),
\end{aligned}
$$

separating the radial and azimuthal parts of the deviations [13]. This is the same form that was used to describe vortex-magnon scattering with ferromagnet with easy-plane anisotropy [14], and it can be shown to be exact in the linear approximation when considering magnons, fully taking into account the nonlocal magnetostatic field. Now the edge contribution to the potential can be evaluated by integration of $\Phi_{s}$ in Eq. (5) using the above ansatz with $-\psi(r, \chi, t)$ as the edge surface charge density. First the integration 
over $z^{\prime}$ in the definition of the magnetostatic potential is done by assuming that the magnetization is uniform along the ring axis, which should be a good approximation for $L \ll R_{i, o}$. Then partial differentiation with respect to $r$ gives the edge-charge magnetostatic field as

$$
H_{r}= \pm R_{i, o} L g(r) \sin (m \chi+\omega t) F\left(r, R_{i, o}\right),
$$

where, as before, the sign corresponds to the inner and outer edges of the ring. The function $F$ is defined by the angular integral

$$
F\left(r, R_{i, o}, m\right)=\int_{0}^{2 \pi} \frac{\left(R_{i, o} \cos \alpha-r\right) \cos m \alpha}{\left(R_{i, o}^{2}+r^{2}-2 R_{i, o} r \cos \alpha\right) \sqrt{R_{i, o}^{2}+r^{2}-2 R_{i, o} r \cos \alpha+L^{2} / 4}} d \alpha
$$

and $\alpha=\chi^{\prime}-\chi$. The edge charge magnetostatic energy is obtained by using $H_{r}$ in Eq. (3) and integration of Eq. (4). The resulting angular integration is trivial leading to a factor of $2 \pi$ for $m=0$ or $\pi$ for $m \neq 0$ with a remaining radial integration in this expression,

$$
\begin{aligned}
W_{\text {edge }} & =\frac{I_{m} L^{2} \pi}{2}\left[R_{o} g\left(R_{o}\right) \int_{R_{i}}^{R_{o}} g(r) F\left(r, R_{o}\right) r d r-\right. \\
& \left.-R_{i} g\left(R_{i}\right) \int_{R_{i}}^{R_{o}} g(r) F\left(r, R_{i}\right) r d r\right]
\end{aligned}
$$

where $I_{m}=2$ for $m=0$ and $I_{m}=1$ for $m \neq 0$.

The effective boundary conditions are obtained through a variation of the energy as was done previously $[8,13]$ for the case of the vortex-state magnetic disk. First the inhomogeneous exchange energy containing the gradient operator in Eq. (3) can be integrated over $z$ and $\chi$ to obtain this energy as a radial integral,

$$
W_{\mathrm{ex}}=A L I_{m} \pi / 2 \int_{R_{i}}^{R_{o}}\left[g_{r}^{2}+f_{r}^{2}+\frac{1}{r^{2}}\left(g^{2}+f^{2}\right)\right] r d r
$$

where the subscripts indicate differentiation. A careful variation of the energy, $\delta \mathrm{W} / \delta g$, will also result in «surface» terms evaluated at $r=R_{i, o}$ as well as terms leading to the Landau-Lifshitz equation. Included in the surface terms will be the $r$-derivative, as a result of integration by parts, as well as the edge contribution to the magnetostatic energy to give the effective boundary conditions at the inner and outer edges,

$$
\left.\frac{d g}{d r}\right|_{r=R_{o, i}} \pm \Lambda_{i, o} g\left(R_{i, o}\right)=0,
$$

where the upper sigh is used at the outer edge and the lower sign is used at the inner edge, and

$$
\Lambda_{i, o}=\frac{L}{2 \pi l_{0}^{2}} \int_{R_{i}}^{R_{o}} F\left(r, R_{o, i}\right) r d r .
$$

The dynamic equations for the small deviations from the vortex state are obtained from the variation of the energy including both the exchange and magnetostatic energy. Now, however, the magnetostatic terms arise from the volume charge only since the edges were accounted for in the boundary conditions. This gives the Landau-Lifshitz equations satisfied by $g$ and $f$. Linearizing these equations we obtain the set of coupled equations

$$
\begin{gathered}
\Omega f=-l_{0}^{2} \nabla^{2} g+\hat{U}, \\
\Omega g=-l_{0}^{2} \nabla^{2} f+\left(1-\frac{l_{0}^{2}}{r^{2}}\right) f,
\end{gathered}
$$

where $\Omega=\omega / 4 \pi \gamma M_{S}$ is a dimensionless magnon frequency, $l_{0}=\sqrt{A / 4 \pi M_{S}^{2}}$ is exchange length. The local part of the magnetostatic field is contained in the Schrödinger-like operators in the first terms of Eqs. (13), (14), and the volume magnetostatic effects are included in the integral operator,

$$
\hat{U}=-\frac{1}{4 \pi M_{s}} \frac{\partial \Phi_{v}}{\partial r} .
$$

For the parameters applicable here, an approximate solution of Eq. (14) is $f=\Omega g$, which results in the equation satisfied by $g$ :

$$
\nabla^{2} g+\frac{\Omega^{2}}{l_{0}^{2}} g=-\frac{1}{4 \pi M_{s}^{2} l_{0}^{2}} \frac{\partial \Phi_{v}}{\partial r} .
$$

In the following it is assumed that the left-hand side of Eq. (16) is small, allowing perturbation techniques to be applied. For all modes the zeroth order solution without the magnetostatic term are known for any boundary condition as the sum of Bessel functions

$$
g_{0}(k, r)=J_{m}(k r)+\sigma Y_{m}(k r),
$$


where $k$ is the wave number and $\sigma$ is a constant that will be determined from the boundary conditions at the inner and outer edges. Now a perturbation technique is developed to obtain the frequency in terms of the nonlocal magnetostatic operators, with $\Phi_{v} / 4 \pi M_{s} \approx L / R_{o}$ and $k l_{0}$ being small parameters. Then from Eq. (16) the following expression for the frequency can easily be obtained:

$$
\Omega^{2}\left\langle g_{0}^{2}\right\rangle=\left(k l_{0}\right)^{2}-\left\langle\frac{g_{0}}{4 \pi M_{s}^{2}} \frac{\partial \Phi_{v}}{\partial r}\right\rangle,
$$

where the brackets indicate the integration, $\langle\ldots\rangle=2 \pi \int_{R_{i}}^{R_{o}} \ldots r d r$. Integration by parts gives the simple expression for the frequency

$\left(\Omega^{2}-\Omega_{0}^{2}\right) \int_{R_{i}}^{R_{o}} g_{0}^{2} r d r=\frac{1}{4 \pi} \int_{R_{i}}^{R_{o}} d r \int_{R_{i}}^{R_{o}} d r^{\prime} \rho(r) g_{0}(k r) F\left(r, r^{\prime}, m\right)$,

where $\Omega_{0}=k l_{0}$ and $\rho(r)=d\left(r g_{0}\right) / d r$.

Next we obtain the values of $k$ and $\sigma$ by substitution of this form of $g_{0}$ into Eq. (11) at the outer and inner edges. To get the expression for $\sigma$ in terms of the wave number, it is enough to consider the condition at $r=R_{0}$,

$$
\sigma=-\frac{k J_{m}^{\prime}\left(k R_{o}\right)+\Lambda_{o} J_{m}\left(k R_{o}\right)}{k Y_{m}^{\prime}\left(k R_{o}\right)+\Lambda_{o} Y_{m}\left(k R_{o}\right)} .
$$

Here and in the next equation we used the notation $J_{m}^{\prime}=d J_{m}(z) / d z, Y_{m}^{\prime}=d Y_{m}(z) / d z$. Notice that the denominator of Eq. (20) can have zeros resulting in discontinuities of this function, which will be noted later. When Eq. (20) is used in Eq. (11) at the inner edge, $R_{i}$ we obtain the equation giving the wave numbers,

$$
\begin{gathered}
H(k) \equiv\left[k J_{m}^{\prime}\left(\rho_{i}\right)-\Lambda_{i} J_{m}\left(\rho_{i}\right)\right] \times \\
\times\left[k Y_{m}^{\prime}\left(\rho_{o}\right)+\Lambda_{o} J_{m}\left(\rho_{o}\right)\right]-\left[k J_{m}^{\prime}\left(\rho_{o}\right)+\Lambda_{o} J_{m}\left(\rho_{o}\right)\right] \times \\
\times\left[k Y_{m}^{\prime}\left(\rho_{i}\right)-\Lambda_{i} J_{m}\left(\rho_{i}\right)\right]=0,
\end{gathered}
$$

where brevity the notation $\rho_{i}=k R_{i}, \rho_{o}=k R_{0}$ is used. Numerical solution of Eq. (21) gives the values of the wave numbers, and owing to the oscillatory property of the Bessel function, there are an infinite number of zeros, with the smallest $k$ corresponding to the lowest frequency. Here we will only consider the first two or three zeros of the $m=0,1,2$ modes, which are illustrated as a plot of $H(k)$ versus $k$ in Fig. 1 for the $m=0$ mode $R_{o}=200 \mathrm{~nm}, R_{i}=100 \mathrm{~nm}$ ring of thickness $L=50 \mathrm{~nm}$. The values of $k$ are then used in Eq. (20) to calculate the parameter $\sigma$, both of which are shown in Fig. 2 as a function of $R_{i}$ with

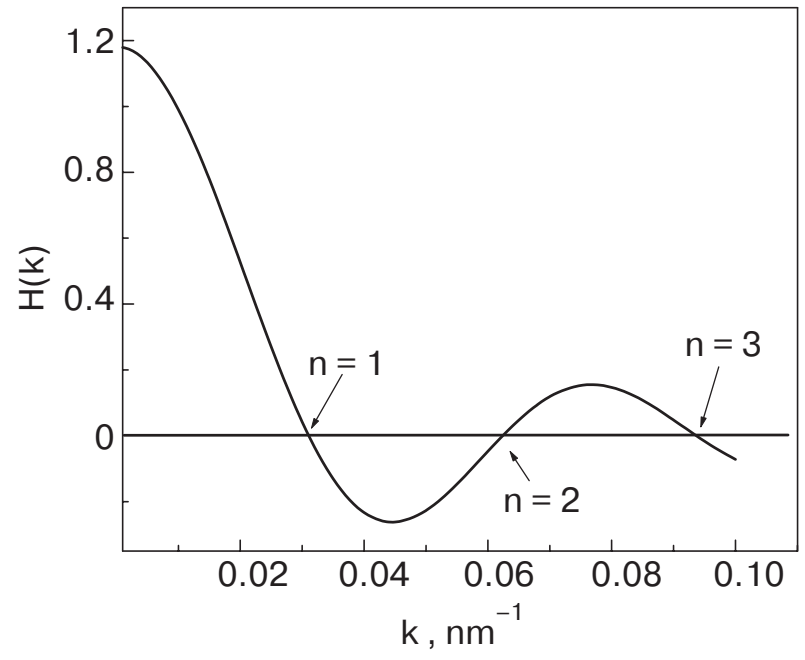

Fig. 1. The left-hand side of Eq. (21), represented by $H(k)$ as a function of $k$, showing the first three zeros, $n=1,2,3$.

$R_{O}=200 \mathrm{~nm}$ and $L=50 \mathrm{~nm}$. Notice that $k$ is a smoothly increasing function of $R_{i}$, but the parameter $\sigma$ is discontinuous owing to the nature of the boundary conditions and Bessel functions.

The nature of the radial eigenfunction for the $R_{O}=200 \mathrm{~nm}, R_{i}=100 \mathrm{~nm}$ ring of thickness $L=50 \mathrm{~nm}$ is illustrated in Fig. 3. Also these radial solutions are approximately pole-free, implying that the zeros are very close to the inner and outer edges, and they closely resemble the Bessel functions $J_{m}(k r)$. Moreover, as the eigenvalue $n$ increases, there are additional interior nodes, and the number of interior nodes is $n-1$.

Finally, the values of $k$ and $\sigma$ are used in Eq. (19) to calculate the mode frequencies for the $R_{O}=200 \mathrm{~nm}$ ring of thickness $L=50 \mathrm{~nm}$, and these results are shown in Fig. 4 for the first two radial modes and the first three azimuthal modes. It is seen that there is a

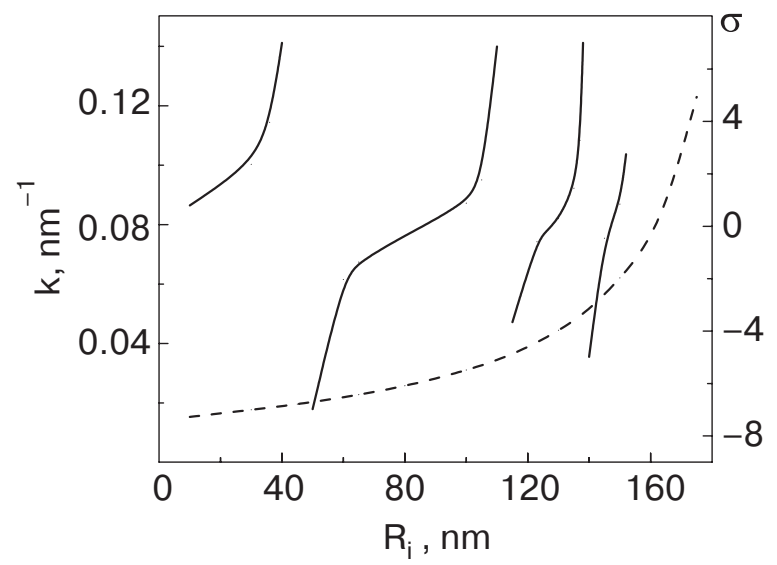

Fig. 2. The wave number $k_{1}$ (dashed curve) and $\sigma$ (solid curve) versus the inner ring radius, $R_{i}$ for the $m=0$ mode. Here $R_{O}=200 \mathrm{~nm}$ and $L=50 \mathrm{~nm}$. 


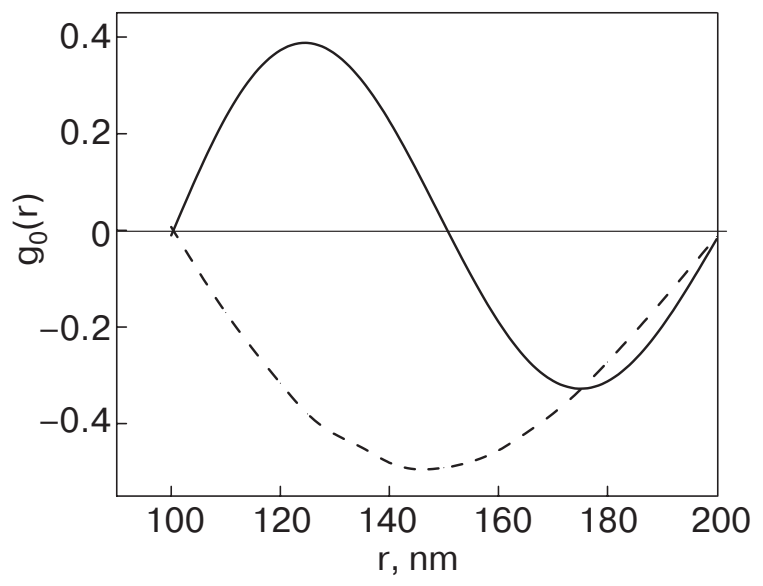

Fig. 3. The zeroth order radial eigenstate for the $m=0$ azimuthal mode showing the $n=1$ (dashed) and $n=2$ (solid) modes.

set of modes corresponding to the azimuthal number $m$ for each value of $n$, starting with the lowest frequencies for $n=1$. It is also interesting to observe that the $m=0$ mode has the highest frequency for every value of $n$ considered here, and the frequency decreases with increasing $m$. This effect referred to as negative dispersion has also been recently observed [15] in the magnon mode spectrum for the vortex-state disk. For the case of wide rings $\left(R_{i} \approx 40 \mathrm{~nm}\right)$ a very rich spectrum is seen in Fig. 4, with the frequency of the $n=1, m=0$ mode close to the $n=2, m=2$ mode. For narrower rings the splitting of the azimuthal modes decreases and the $n=1, n=2$ becomes distinct. For $R_{i} \approx 130 \mathrm{~nm}$ the azimuthal mode splitting becomes less than $1 \mathrm{GHz}$, which is the approximate resolution of time-resolved Kerr microscopy.

In conclusion, there is a rich spectrum of magnon modes excited in Permalloy vortex-state rings that can be classified according to radial and azimuthal $(n, m)$

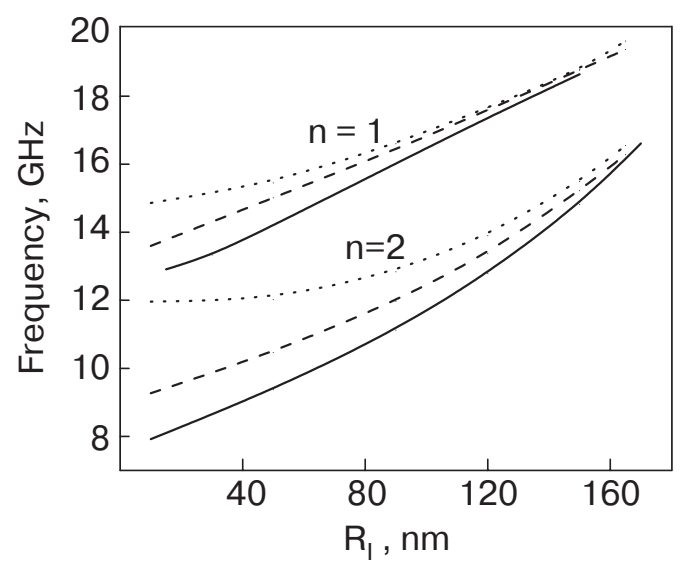

Fig. 4. Frequency versus inner ring radius, $R_{i}$ for the $n=1$ and $n=2$ radial modes. Azimuthal modes are $m=0$ (dots), $m=1$ (dashed) and $m=2$ (solid) for the $R_{O}=200 \mathrm{~nm}$ and $L=50 \mathrm{~nm}$ ring. eigenvalues. The $m=1$ mode can be excited by an in-plane pulse, which has been shown to reverse the curling direction in the vortex-state ring. For this reason, the $m=1$ mode can possibly have effects on the dynamics of vortex switching. The $m=0$ modes can be excited by an out-of-plane pulse of a magnetic field. Other modes not considered here are the higher frequency modes with additional radial modes $n>2$ as well as the higher $m$ modes. The frequency of any mode can be obtained from numerical solution and integration of Eqs. (17)-(19) exhibiting a structure similar to Fig. 4.

This work was supported by the National Science Foundation Grant number DMR-9972507.

1. W.J. Gallagher, S.S.P. Parkin, Y. Lu, X.P. Bian, A. Marley, K.P. Roche, R.A. Altman, S.A. Rishton, C. Jahnes, T.M. Shaw, and G. Xiao, J. Appl. Phys. 81, 3741 (1997).

2. T. Shinjo, T. Okuno, R. Hassdorf, K. Shigeto, and T. Ono, Science 289, 930 (2000).

3. J.P. Park, P. Eames, D.M. Engebretson, J. Berezovsky, and P.A. Crowell, Phys. Rev. B67, 020403(R) (2003); C.E. Zaspel, B.A. Ivanov, J.P. Park, and P.A. Crowell, Phys. Rev. B72, 024427 (2005).

4. J. Rothman, M. Klaui, L. Lopez-Diaz, C.A.F. Vaz, A. Bleloch, J.A.C. Bland, Z. Cui, and R. Speaks, Phys. Rev. Lett. 86, 1098 (2001).

5. U. Welp, V.K. Vlasko-Vlasov, J.M. Miller, N.J. Zaluzec, V. Metlushko, and B. Ilic, Phys. Rev. B68, 054408 (2003).

6. F.J. Castano, C.A. Ross, C. Frandsen, A. Eilez, D. Gil, H.I. Smith, M. Redjdal, and F.B. Humphrey, Phys. Rev. B67, 184425 (2003).

7. M. Klaui, C.A.F. Vaz, J.A.C. Bland, W. Wernsdorfer, G. Faini, E. Cambril, L.J. Heyderman, F. Nolting, and U. Rudiger, Phys. Rev. Lett. 94, 106601 (2005).

8. B.A. Ivanov and C.E. Zaspel, Phys. Rev. Lett. 94, 027205 (2005).

9. R. Zivieri and F. Nizzoli, Phys. Rev. B71, 014411 (2005).

10. K. Yu. Guslienko, W. Scholz, R.W. Chantrell, and V. Novosad, Phys. Rev. B71, 144407 (2005).

11. M. Buess, R. Hollinger, T. Haug, K. Perzlmaier, U. Krey, D. Pescia, M.R. Scheinfein, D. Weiss, and C.H. Back, Phys. Rev. Lett. 93, 077207 (2004).

12. W. Xu, D.B. Watkins, L.E. DeLong, K. Rivkin, J.B. Ketterson, and V.V. Metlushko, J. Appl. Phys. 95, 6645 (2004).

13. B.A. Ivanov and C.E. Zaspel, Appl. Phys. Lett. 81, 1261 (2002).

14. B.A. Ivanov, H.J. Schnitzer, F.G. Mertens, and G.M. Wysin, Phys. Rev. B58, 8464 (1998).

15. M. Buess, T.P.J. Knowles, R. Hollinger, T. Haug, U. Krey, D. Weiss, D. Pescia, M.R. Scheinfein, and C.H. Back, Phys. Rev. B71, 104415 (2005). 